\title{
ORTHO AMINO CYANO AROMATIC COMPOUNDS AS PRECURSOR FOR THY SYNTHESIS OF SOME NOVEL HETEROCYCLIC COMPOUNDS
}

\author{
V.S.Rao* ${ }^{a}$, S.V.S. Arun Kumar Gupta ${ }^{a}$ and B.S.Reddy ${ }^{b}$ \\ a: Department of Chemistry, Birla Institute of Technology \& Science, Pilani, Rajastahn, India. \\ B: Suven Pharmaceuticals. Hyderabad.
}

\begin{abstract}
Pyrimidine-4(1H)-ones from 2-amino-3-cyano aromatic compounds were prepared in the presence of formic acid under strong acid catalyst. The intermediates and the final products structures were confirmed by spectral and elemental data.
\end{abstract}

\section{Introduction:}

2-amino-3-cyano aromatic compounds are used as a building blocks for the synthesis of fused pvrimidine$4(1 \mathrm{H})$-ones, which are intermediates in the synthesis of antipyretics, diuretics, anticonvulsants, sedatives. coronary dilators and anti-cancer agents ${ }^{1-4}$. Historically quinazolines- $4(1 \mathrm{H})$-ones have been prepared by Neimentowski reaction of anthranilic acids ${ }^{5}$. Other literature methods were cyclisatioon of ortho eaters ${ }^{6}$, Gold's reagents ?, ethoxymethylenemalononitrile with 6 -amino benzaxamides ${ }^{8}$, amonolysis of benzoxazines ${ }^{9}$, synthesis of quinazoline derivatives via, palladium catalyzed reductive cyclisation methods ${ }^{10}$. Literature reveals that ortho cyano amino compounds are often more readily accessible synthetic targets than the corresponding anthranilic acids

\section{Synthesis and Results:}

For the first time, we are reporting a new cyclisation method on 2-amino-3-cyano pyridines, pyrazolines and pyridones systems, which affords good yields of pyrido-(2,3-d)-pyrimidine-4(1H)-ones.(II), Pyrazolo(3,4-d)-pyrimidine-4(7H)-ones (IV) and pyrido-(2,3-d)-pyrimidine-4.7-(1H)-diones (VI) respectively. The advantage of this method is simpler experimental conditions easy isolation procedure, shorter reaction times and delivery of the desired product in good purity with quantitative yields ${ }^{-14}$.
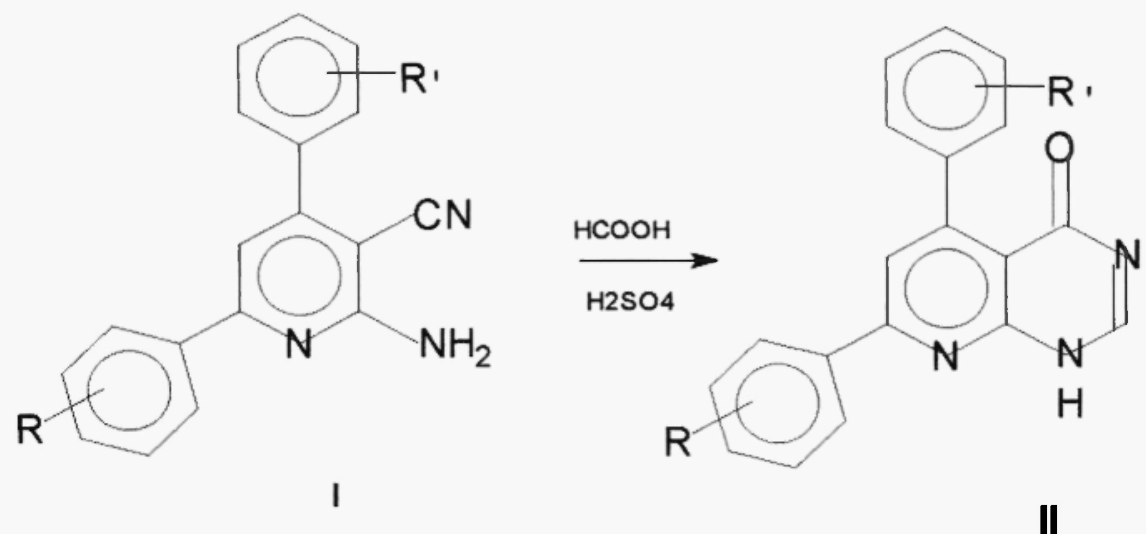


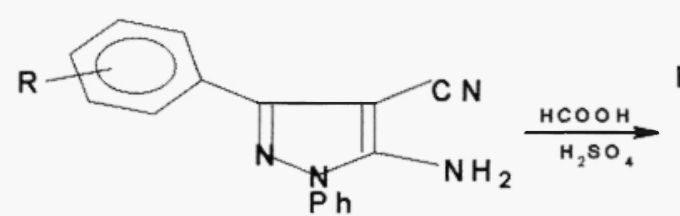<smiles>[R]c1cccc(-c2c(C#N)c(N)[nH]c(=O)c2C#N)c1</smiles>

v

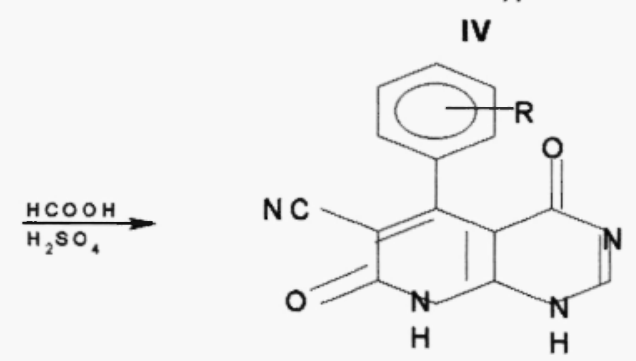

V I

Cyclisation of 2-cyano-3-amino-5,7-diaryl pyridines (I), 2-cyano-3amino-5-aryl pyrazoles (III) , 25dicayno-3-amino-4-aryl-pyridine-6-ones $(V)$ with formic acid in the presence of a trace amount of sulfuric acid at $110 \mathrm{C}$ doe $25 \mathrm{~min}$, gave compound II, IV, VI respectively. The structure of the compound were established on the basis of IR, PMR and Mass spectral data and the result of elemental analysis (see table-I). The PMR spectra of compounds II,IV and VI are quite interesting in that the protons $\mathbf{N}-\mathbf{H}$ and vinyl protons are observed in the range of 7.5 and 8.3 ppm respectively ${ }^{15}$.

The synthesis of the starting compounds I, III and V was carried out according to the literature procedure. The reaction is believed to proceed via the formation of an amide intcrmediate. This intermediates is isolated and synthesized on a different route. Compounds on further cyclisation under the same experimental conditions gave II, IV and VI, which was found to be identical with compounds obtained earlier.<smiles>[R]c1cc([R])c(C(=N)OC=O)c(N)n1</smiles><smiles></smiles>

SCHEME- II 
TABLE-1: Physical , $\mathrm{C}, \mathrm{H}, \mathrm{N}$ analysis

\begin{tabular}{|c|c|c|c|c|c|c|c|c|}
\hline \multirow[t]{2}{*}{$\begin{array}{l}\text { Compd. } \\
\text { No. }\end{array}$} & \multirow[t]{2}{*}{$R$} & \multirow[t]{2}{*}{$\overline{R^{\prime}}$} & \multirow[t]{2}{*}{$\begin{array}{l}\text { Yield } \\
\text { (\%) }\end{array}$} & \multirow[t]{2}{*}{$\begin{array}{l}\text { M.P. } \\
\text { (C) }\end{array}$} & \multirow[t]{2}{*}{$\begin{array}{l}\text { Mol.for } \\
\text { (Mol.wt) }\end{array}$} & \multicolumn{3}{|c|}{$\begin{array}{c}\text { Analysis found (Calcd.) } \\
(\%)\end{array}$} \\
\hline & & & & & & $\mathrm{C}$. & $\mathrm{H}$ & $\bar{N}$ \\
\hline II a. & $\mathrm{CH}_{3}$ & $\mathrm{H}$ & 76 & $230-31$ & $\begin{array}{c}\mathrm{C}_{8} \mathrm{H}_{7} \mathrm{~N}_{3} \mathrm{O} \\
(161)\end{array}$ & $\begin{array}{c}59.60 \\
(59.62)\end{array}$ & $\begin{array}{c}4.34 \\
(4.29)\end{array}$ & $\begin{array}{c}26.08 \\
(26.10)\end{array}$ \\
\hline b. & $\mathrm{C}_{0} \mathrm{H}_{5}$ & $H$ & 78 & $199-202$ & $\begin{array}{c}\mathrm{C}_{13} \mathrm{H}_{2} \mathrm{~N}_{3} \mathrm{O} \\
(223)\end{array}$ & $\begin{array}{c}69.89 \\
(69.95)\end{array}$ & $\begin{array}{l}4.03 \\
(3.99)\end{array}$ & $\begin{array}{l}18.81 \\
(18.84)\end{array}$ \\
\hline c. & $\mathrm{C}_{0} \mathrm{H}_{5}$ & $\mathrm{C}_{0} \mathrm{H}_{5}$ & 75 & $210-12$ & $\begin{array}{c}\mathrm{C}_{10} \mathrm{H}_{13} \mathrm{~N}_{3} \mathrm{O} \\
(301)\end{array}$ & $\begin{array}{r}75.69 \\
(75.74)\end{array}$ & $\begin{array}{r}4.29 \\
(431)\end{array}$ & $\begin{array}{r}13.87 \\
(13.95)\end{array}$ \\
\hline d & $4-\mathrm{ClC}_{6} \mathrm{H}_{4}$ & $\mathrm{Cor}_{5}$ & 78 & $216-18$ & $\begin{array}{c}\mathrm{C}_{19} \mathrm{H}_{12} \mathrm{~N}_{3} \mathrm{OCCl} \\
(335.5)\end{array}$ & $\begin{array}{c}67.89 \\
(67.94)\end{array}$ & $\begin{array}{c}3.50 \\
(3.57)\end{array}$ & $\begin{array}{c}12.48 \\
(12.51)\end{array}$ \\
\hline e. & $\mathrm{C}_{6} \mathrm{H}_{5}$ & $2-\mathrm{Cl}_{-} \mathrm{C}_{6} \mathrm{H}_{5}$ & 86 & $240-42$ & $\begin{array}{c}\mathrm{C}_{19} \mathrm{H}_{12} \mathrm{~N}_{3} \mathrm{OCl} \\
(3335.5)\end{array}$ & $\begin{array}{l}67.90 \\
167.94\end{array}$ & $\begin{array}{c}3.56 \\
(3.57)\end{array}$ & $\begin{array}{l}12.44 \\
(12.51)\end{array}$ \\
\hline iv a. & H & $\mathrm{C}_{0} \mathrm{H}_{5}$ & 77 & $216-18$ & $\begin{array}{c}\mathrm{C}_{17} \mathrm{H}_{12} \mathrm{~N}_{4} \mathrm{O} \\
(288)\end{array}$ & $\begin{array}{c}70.80 \\
(70.82)\end{array}$ & $\begin{array}{c}4.15 \\
(4.16)\end{array}$ & $\begin{array}{l}19.40 \\
(19.44)\end{array}$ \\
\hline b. & H & $4 \mathrm{ClC}_{6} \mathrm{H}_{4}$ & 79 & $232-34$ & $\begin{array}{c}\mathrm{C}_{11} \mathrm{H}_{11} \mathrm{~N}_{4} \mathrm{O} \\
(322.5)\end{array}$ & $\begin{array}{c}63.20 \\
(63.24)\end{array}$ & $\begin{array}{l}3.45 \\
(341)\end{array}$ & $\begin{array}{l}17.30 \\
(17.36)\end{array}$ \\
\hline c. & H & $3-\mathrm{NO}_{2} \mathrm{C}_{6} \mathrm{H}_{4}$ & 71 & 240-42 & $\begin{array}{c}\mathrm{C}_{17} \mathrm{H}_{11} \mathrm{~N}_{8} \mathrm{O}_{3} \\
(328)\end{array}$ & $\begin{array}{c}62.15 \\
(62.17)\end{array}$ & $\begin{array}{c}3.30 \\
(3.35)\end{array}$ & $\begin{array}{l}19.55 \\
(1950)\end{array}$ \\
\hline d. & H & $2-\mathrm{ClC}_{6} \mathrm{H}_{4}$ & 81 & $222-23$ & $\begin{array}{c}\mathrm{C}_{17} \mathrm{H}_{11} \mathrm{~N}_{4} \mathrm{OCl} \\
(330)\end{array}$ & $\begin{array}{r}61.79 \\
(61.81)\end{array}$ & $\begin{array}{l}3.29 \\
(3.33)\end{array}$ & $\begin{array}{c}16.91 \\
(1696)\end{array}$ \\
\hline e. & H & $4 \mathrm{CH} 3 \mathrm{C} 6 \mathrm{H} 4$ & 80 & $198-99$ & $\begin{array}{c}\mathrm{C}_{18} \mathrm{H}_{1}, \mathrm{~N}_{4} \mathrm{O} \\
(310)\end{array}$ & $\begin{array}{c}69.65 \\
(69.66)\end{array}$ & $\begin{array}{l}4.45 \\
(4.51)\end{array}$ & $\begin{array}{r}18.10 \\
(18.06)\end{array}$ \\
\hline VI a. & H & $\mathrm{C}_{6} \mathrm{H}_{5}$ & 75 & $211-12$ & $\begin{array}{c}\mathrm{C}_{13} \mathrm{H}_{9} \mathrm{~N}_{3} \mathrm{O}_{2} \\
(239)\end{array}$ & $\begin{array}{c}65.19 \\
(65.22)\end{array}$ & $\begin{array}{l}3.72 \\
(3.76)\end{array}$ & $\begin{array}{c}17.60 \\
(17.56)\end{array}$ \\
\hline b. & H & $2-\mathrm{ClC}_{6} \mathrm{H}_{4}$ & 61 & $205-06$ & $\begin{array}{c}\mathrm{C}_{13} \mathrm{H}_{8} \mathrm{~N}_{3} \mathrm{O}_{2} \mathrm{Cl} \\
(273.5)\end{array}$ & $\begin{array}{c}56.91 \\
(57.03)\end{array}$ & $\begin{array}{l}2.90 \\
(2.92)\end{array}$ & $\begin{array}{l}15.28 \\
(15.35)\end{array}$ \\
\hline c. & H & $3-\mathrm{NO}_{2} \mathrm{C}_{6} \mathrm{H}_{4}$ & 65 & $234-36$ & $\begin{array}{c}\mathrm{C}_{13} \mathrm{H}_{4} \mathrm{~N}_{4} \mathrm{O}_{4} \\
(284)\end{array}$ & $\begin{array}{c}54.96 \\
(54.92)\end{array}$ & $\begin{array}{l}2.77 \\
(2.81)\end{array}$ & $\begin{array}{l}19.80 \\
(1971)\end{array}$ \\
\hline d. & $H$ & $4 \mathrm{CH}_{3} \mathrm{C}_{6} \mathrm{H}_{4}$ & 67 & $217-19$ & $\begin{array}{c}\mathrm{C}_{14}+\mathrm{H}_{1}, \mathrm{~N}_{3} \mathrm{O}_{2} \\
(253)\end{array}$ & $\begin{array}{c}66.35 \\
(66.39)\end{array}$ & $\begin{array}{r}4.30 \\
(4.34)\end{array}$ & $\begin{array}{l}16.51 \\
(1659)\end{array}$ \\
\hline e. & H & $4 \mathrm{BrC}_{6} \mathrm{H}_{4}$ & 70 & $254-56$ & $\begin{array}{l}\mathrm{C}_{13} \mathrm{H}_{6} \mathrm{~N}_{2} \mathrm{O}_{2} \mathrm{Br} \\
(318)\end{array}$ & $\begin{array}{l}49.00 \\
(49.04)\end{array}$ & $\begin{array}{l}2.46 \\
(2.51)\end{array}$ & $\begin{array}{r}13.16 \\
(13.20)\end{array}$ \\
\hline
\end{tabular}

TABLE - 2: Spectral data.

\begin{tabular}{|c|c|c|}
\hline $\begin{array}{l}\text { Compou } \\
\text { nd No. }\end{array}$ & IR data ( $\max$ ) & PMR ( ppm) \\
\hline $\begin{array}{ll}\text { II } & \text { a. } \\
& \text { c. } \\
& \text { d. } \\
\text { IV } & \text { a. } \\
& \text { b. } \\
\text { VI } & \text { a. } \\
& \text { d. }\end{array}$ & $\begin{array}{l}1645(C=O), 1590(C=N) \\
3450(N-H) \\
1640(C=O), 1580(C=N) \\
3454(N-H) \\
1640(C=O), 1575(C=N) \\
3380(N-H) \\
1630(C=C), 1680(C=O) \\
1590(C=N), 3350(N-H) \\
1615(C=C), 1685(C=O) \\
1600(C=N) 3340(N-H) \\
1690(C=O), 1620(C=C) \\
1580(C=N), 3300-3400 \\
(N-H) \\
1685(C=C), 1690(C=O) \\
1590(C=N), 3350-3400 \\
(N-H)\end{array}$ & 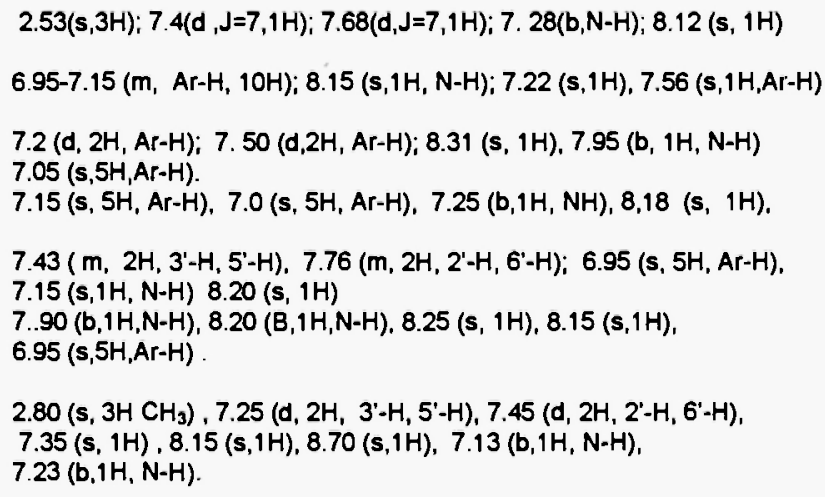 \\
\hline
\end{tabular}




\section{Experimental Section:}

Melting points were determined on Mel.Temp and are uncorrected. IR spectra were recorded in $\mathrm{KBr}$ on Perkin-Elmer spectrometer, PMR were recorded in DMSO-d6 and CDCl3 on Varian-Gemeini 200 spectrometer.

General procedure for the preparation of fused Pyrimidine-4(IH)-ones (II, IV \& VI):

A mixture of 2-amino-3-cyano -aromatic compound ( 2 mmoles) was added in portion wise over $1 \mathrm{hr}$ to a mildly reflecting mixture of $88 \%$ formic acid and sulfuric acid $(1.0 \mathrm{gm})$. After $15 \mathrm{~min}$, the mixture was allowed to cool to $60 \mathrm{C}$, poured into crushed ice ( $100 \mathrm{gms})$ and allowed to stand for $15 \mathrm{~min}$. The resulting precipitate was collected and washed well with water. Drying to constant weight provided II, IV \&VI as an off white solid (80\%).

Acknowledgments: One of the authors (SVSAKG) is thankful to Prof.Venkateswaran, Director, BITS, Pilani (Raj), India. for providing the necessary research facilities.

\section{References:}

1. S.S.F.Burni, A.Castanzo, G.Guerrini, P.M.Ajello, G.Iavsrone and C.Martini, Eur.J.Med.Chem. 27. 985 (1992).

2. M.F.Hasan. A.M.Mounir,, I.Sallem,J.M.Abdul Rahman and A.E.Mohammed, Hetero cycles, $\underline{38}$ 57 (1994).

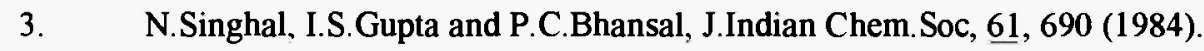

4. A.K.Sharma, A.K.Yadav and Lalit Prakash, Ind.J.Chemistry, 34B, 740 (1990).

5. V.Neimentoskii. J.Pract.Chem, 51, 564 (1895); W.L.F.Armarego, J.Appl.Chem, 11, 70 (1961).

6. M.K.Mckee, R.L.McKee and R.W.Bost, J.Am.Chem.Soc, $\underline{69}_{2} 184$ (1947).

7. K.Nagahara, K.Takagi and T.Ueda, Chem. Pharm.Bull, 24, 310 (1976).

8. J.T.Gupton, K.F.Correia, and B.S.Foster, Synth.Comm, 16, 365, (1986).

9. M.T.Bogert and W.F.Hard, J.Am.Chem.Soc, $2 \underline{8}, 94$ (1906).

10. M.Akazome, J.Yamamotto, T.Kordo and Y.Wantanhe, J.Organomet.Chem, 494, (1995).

11. M.Shah, P.Patel.S.Korgaokar and H.Parekh. Indian.J.Chem.SOc, 35B, 1282 (1996).

12. U.Hanefeld, V.W.Rees. A.J.P.White and D.J.Williams, J.Chem>Soc, Per.Tran-1,1546, (1996).

13. A.H.H.Elghandour, M.K.Abrahim, F.M.M.Ali and S.M.M.Elshikh, Indian.J.Chem, $\underline{36 \mathrm{~B}}, \mathbf{7 9}$ (1997).

14. G.A.Roth and J.J.Tai, J.Hetrtocyclic.Chem, 33, 2051 (1996).

15. U.T.Bhale Rao and A.Krishnaiah, Indian.J.Chem, 34B, 587 (1995).

\section{Received on December 20, 1998}

